\title{
Parameter estimation of measured channels in mobile MIMO OFDM system
}

\author{
Mihai Enescu*1, Markus Herdin ${ }^{* *}$, Timo Roman ${ }^{*}$ and Visa Koivunen* \\ \{mihai, troman, visa\}@wooster,hut.fi, markus.herdin@nt.tuwien.ac.at \\ *SMARAD CoE, Signal Processing Laboratory, Helsinki University of Technology \\ P.O. Box 3000, FIN-02015 HUT, Finland \\ ${ }^{* *}$ Institute of Communications and Radio-Frequency Engineering, Vienna University of Technology \\ Gusshausstrasse 25/389, A-1040 Wien, Austria
}

\begin{abstract}
In this paper the feasibility of adaptive channel parameter estimation method for measured MIIMO channels is studied. Our investigations are based on building a state-space model for the MMO OFDM transmission, that accounts also for the spatial correlation at transmitter and receiver antenna arrays. Kalman filter is then applied to estimate and track the time-varying channels. Correlation parameters are also estimated from the received data. Our simulations are based on measured MIMO channels and show that reliable channel estimation can be performed under realistic conditions.
\end{abstract}

\section{INTRODUCTION}

Radio spectrum is a scarce resource in wireless communication. High spectral efficiency is therefore a major goal of future mobile wireless communications systems. Multiple-input multipleoutput (MIMO) techniques are promising candidates for such systems [12] since they additionally utilize the spatial domain by doing multiplexing and therefore obtain much higher capacities than conventional single-input single-output (SISO) systems. In order to enjoy improved capacity, the scattering environment needs to be rich, where several different propagation paths exist between the transmitter and the receiver. The capacity gain depends on the available channel information at either the receiver or transmitter, on the signal to noise ratio (SNR), and on the spatial correlation [7], [14].

In realistic scenarios, both uncorrelated and correlated channels are encountered. An often used approach to model correlated MIMO channels is the I-METRA model, also known under the name "Kronecker model". There, the MIMO channel is modeled by the transmit and receive correlation matrix. This means that it is assumed that the transmit correlation matrix is independent on the receive correlation matrix [6], [9], [14].

Accurate channel estimation plays an important role in achieving the high capacity of MIMO systems. The problem of correlated channel coefficients in a SISO system has been also considered in $[15]$, where the time-varying channel was modeled as a multichannel autoregressive process of order $p$. The state transition matrix has been estimated from the received data by using higher order statistics.

In this paper we investigate the performance of a recursive channel parameter estimation method introduced in [5], when applied to real MIMO measured channels. A state-space model for correlated channels is derived starting from a model for uncorrelated channels. This model includes the correlation matrix into the state variable assuming that the correlation matrix is estimated from the received data.

The rest of the paper is organized as follows. In the next section we describe how correlated channels can be modeled based on uncorrelated channels. In Section 3 we describe the state-space model in the case of correlated channels and in Section 4 we present how

'This work has been supported by the Academy of Finland and Nokia. we can estimate the correlation from the received data. In Section 5 the MIMO channel measurement setup and simulation results are discussed. Finally, Section 6 concludes the paper.

\section{Correlated mimo cilannels}

An often used MIMO channel model is the I-METRA model that is also referred to as "Kronecker" model [9]. This model has been used for simulations in the standardization process of $3 \mathrm{G}$ systems. Assuming a system with $m$ transmit and $n$ receive antennas in a narrowband scenario, the channel matrix $\mathbf{H}$ will be of size $n \times m$. The input-output relationship may then be written as:

$$
\mathbf{r}_{k}=\mathbf{H}_{k} \mathbf{x}_{k}+\mathbf{w}_{k},
$$

where $\mathbf{r}$ is the received signal, $\mathbf{x}$ are the transmitted symbols, $\mathbf{w}$ is additive Gaussian noise, and $k$ is the time index.

Following the I-METRA model, we define a transmit and receive correlation matrix, $\mathbf{R}_{\boldsymbol{T} x}$ and $\mathbf{R}_{\boldsymbol{R x}}$ that have the dimensions $m \times m$ and $n \times n$, respectively. The correlated channel matrix may then be expressed as:

$$
\mathbf{H}^{(c)}=\mathbf{R}_{R x}^{1 / 2} \mathbf{H}^{(i)}\left(\mathbf{R}_{T x}^{T}\right)^{1 / 2}
$$

where $\mathbf{H}^{(c)}$ is the correlated MIMO channel matrix, $\mathbf{H}^{(i)}$ is the MIMO channel matrix with ii.d. Rayleigh distributed entries and matrix square roots may be obtained, e.g. via Cholesky decomposition.

The spatial correlation at the transmitter and the receiver is modeled independently of each other. This approach was justified by the fact that only the immediate surroundings of the antenna array influences the correlation of the antennas, but that the spatial correlation at the other link end does not have an influence on it [3], [9]. However, this is not fully true as it was shown in [2]. Therefore, a new channel model was introduced [16] that includes the Kronecker model as a special case.

It has been demonstrated that for $2 \times 2$ and $3 \times 3$ systems [2], the Kronecker model is rather accurate, but as soon as the number of transmit and receive antennas increases, it becomes inaccurate. For these cases, the Weichselberger model [16] gives much more rigorous results. This model accounts also for the coupling between the transmit and receive antenna elements.

In this paper we use only $2 \times 2$ systerns and therefore limit our considerations to the Kronecker model. For this model, the fult MIMO channel correlation matrix that describes the correlation between all elements of the channel matrix is given by:

$$
\mathbf{R}_{M I M O}=\mathbf{R}_{T x} \otimes \mathbf{R}_{R \boldsymbol{x}} .
$$

A correlated channel matrix $\mathbf{H}^{(c)}$ may be generated using the $n \times$ $m$ matrix $\mathbf{H}^{(i)}$ and a given MIMO correlation matrix $\mathbf{R}_{M I M O}$ as 
follows. The Cholesky decomposition of the positive definite MIMO correlation matrix is:

$$
\mathbf{R}_{M I M O}=\mathbf{C C}^{H}
$$

Using the vec operator, the channel matrix elements can be stacked into a column vector as: $\mathbf{h}=\operatorname{vec}(\mathbf{H})$, where $\mathbf{h}$ has dimension $m n \times$ 1. The correlated channel matrix in column vector form is obtained by:

$$
\operatorname{vec}\left(\mathbf{H}_{k}^{(c)}\right)=\mathbf{C} \operatorname{vec}\left(\mathbf{H}_{k}^{(i)}\right)
$$

which is equivalent to:

$$
\mathrm{h}_{k}^{\mathrm{c}}=\mathbf{C h}_{k}^{i}
$$

where $k$ is the time index. Using a $2 \times 2$ system as an example (Figure 1), the channel vectors from (4) contain the elements:

$$
\mathbf{h}_{k}^{(c, i)}=\left[h_{11, k}^{(c, i)} h_{21, k}^{(c, i)} h_{12, k}^{(c, i)} h_{22, k}^{(c, i)}\right]^{T^{T}}
$$

In case of frequency selective time-varying model with $L$-tap channels in each MIMO branch, the main assumption is that each delayed path component $(1, \ldots, L)$ exhibits a different correlation structure from the other delayed paths. The relationship between correlated and independent channel taps is given by:

$$
\mathbf{h}_{k}^{c}=\overline{\mathbf{C}}_{k}^{i} \text {, }
$$

where $\tilde{\mathbf{C}}$ is a matrix having the structure:

$$
\check{\mathbf{C}}=\left[\begin{array}{ccc}
\mathbf{C}_{1} & \mathbf{0} & \mathbf{0} \\
\mathbf{0} & \ddots & \mathbf{0} \\
\mathbf{0} & \mathbf{0} & \mathbf{C}_{L}
\end{array}\right],
$$

with each $\mathrm{C}_{l}, l=1, \ldots, L$ being the Cholesky decomposition of the corresponding MIMO spatial correlation. The elements in the channel vectors are arranged as:

$$
\mathbf{h}_{k}=\left[h_{11, k}^{(1)} h_{21, k}^{(1)} h_{12, k}^{(1)} h_{22, k}^{(1)} \ldots h_{11, k}^{(L)} h_{21, k}^{(L)} h_{12, k}^{(L)} h_{22, k}^{(L)}\right]^{T} .
$$

To summarize, spatially correlated MIMO channels can be created from i.i.d. MIMO channel matrices [6], [9], [14], [16] by applying a given correlation pattern. In case of frequency selective channels the same is done separately for each tap by assuming that different taps fade independently.

\section{State-variable Model.}

In wireless mobile communication environments characterized by time selectivity, estimation and tracking of channel parameters is crucial for reliable transmission. In the case of linear FIR channels, we can model the transmission using linear state-space formulation. Kalman filter (KF) may then be applied in order to estimate the state vector comprised of the wireless channel coefficients.

We consider the $m$ input - $n$ output frequency selective MIMO OFDM scenario (See Figure 1 for the $2 \times 2$ case). The state-space formulation is the following:

$$
\begin{aligned}
\mathbf{h}_{k+1}^{i} & =\mathbf{A}_{d} \mathbf{h}_{k}^{i}+\mathbf{v}_{k} \\
\mathbf{r}_{k} & =\tilde{\mathbf{X}}_{k} \mathbf{h}_{k}^{i}+\mathbf{w}_{k} .
\end{aligned}
$$

The state vector of size $m n L \times 1$ is defined as $h^{i}=$ $\left[\mathbf{h}_{11}^{i}, \mathbf{h}_{21}^{i}, \cdots, \mathbf{h}_{m n}^{i}\right]^{T}$, with $\mathbf{h}_{r g}^{i}=\left[h_{r s, 1}^{i}, \ldots, h_{r s, L}^{i}\right]^{T}$, and $r s=$ $11, \ldots, m n$. The observation vector $\mathbf{r}_{k}$ is of dimension $n N \times 1$, where $N$ is the number of subcarriers. Typically $N \gg L$. The circulant matrix containing the transmitted OFDM modulated symbols $\overline{\mathbf{X}}_{k}$ is of size $n N \times m n L$ [13]. It admits a left inverse of dimension $m n L \times n N$ which will be denoted by $\tilde{\mathbf{X}}_{k}^{+}$, i.e. $\overline{\mathbf{X}}_{k}^{+} \overline{\mathbf{X}}_{k}=\mathbf{I}$. The state transition matrix $\mathbf{A}_{d}$ is a $m n L \times m n L$ diagonal matrix. State noise $v$ and measurement noise $w$ with covariance matrices $Q$ and $\mathbf{R}$ are assumed to be zero mean circular white Gaussian, mutually uncorrelated and also uncorrelated with the state vector.

Combining equations (6) and (9) leads to the following dynamic model for the spatially correlated channel:

$$
\mathbf{h}_{k+1}^{c}=\mathbf{A}_{\mathrm{d}} \mathbf{h}_{k}^{c}+\overline{\mathbf{C}}_{k},
$$

under the condition $\mathbf{A}_{d}=a \mathbf{I},|a|<1$.

As in [10] we consider that the first-order AR model approximation is enough to capture the channel dynamics over time. There may also exist one-lag spatial correlation between the taps. Consequently, the state transition matrix is not diagonal, and we will denote it by A. Combining equations (6) and (9) leads to the following dynamic model for the spatio-temporal correlated channel:

$$
\mathbf{h}_{k+1}^{c}=\mathbf{A}_{c} \mathbf{h}_{k}^{c}+\tilde{\mathbf{C}} \mathbf{v}_{k},
$$

where $\mathbf{A}_{c}=\check{\mathbf{C}} \mathbf{A} \check{\mathbf{C}}^{-1}$. The measurement equation remains:

$$
\mathbf{r}_{k}=\check{\mathbf{X}}_{k} \mathbf{h}_{k}^{\mathrm{c}}+\mathbf{w}_{k} \text {. }
$$

Using the state-space model defined by the set of equations (12) and (13), KF can be applied to estimate the state. The update of the covariance matrix of the state prediction error requires the channe] correlation matrix and the state noise covariance:

$$
\mathbf{P}_{(k \mid k-1)}=\mathbf{A}_{c} \mathbf{P}_{(k-1 \mid k-1)} \mathbf{A}_{c}^{H}+\bar{C} Q \bar{C}^{H} .
$$

\section{ESTIMATION}

As noted previously, the correlation matrix $\overline{\mathbf{C}}$ is needed in $\mathrm{KF}$ equations. In fact, we do not need to know specifically the correlation matrix, but the structure $\tilde{\mathrm{C}} Q \tilde{C}^{H}$. The method presented here to estimate $\tilde{\mathrm{C}} \tilde{\mathrm{C}}^{H}$ is based on adaptively updating a quantity based on the received data, on the transmitted symbols and on the state transition matrix. The technique is based on investigating the following quantity:

$$
\begin{aligned}
\mathbf{B}_{1}^{k}= & E\left[\left(\tilde{\mathbf{X}}_{k}^{+} \mathbf{r}_{k}-\mathbf{A}_{c} \tilde{\mathbf{X}}_{k-1}^{+} \mathbf{r}_{k-1}\right)\right. \\
& \left.\left(\tilde{\mathbf{X}}_{k}^{+} \mathbf{r}_{k}-\mathbf{A}_{c} \tilde{\mathbf{X}}_{k-1}^{+} \mathbf{r}_{k-1}\right)^{H}\right]
\end{aligned}
$$

We will first consider the term $\mathbf{S}_{k}=\tilde{\mathbf{X}}_{k}^{+} \mathbf{r}_{k}-\mathbf{A}_{c} \overline{\mathbf{X}}_{k-1}^{+} \mathbf{r}_{k-1}$. Using the measurement equation (13), $S_{k}$ can be further written as:

$$
\begin{aligned}
\mathbf{S}_{k} & =\tilde{\mathbf{X}}_{k}^{+} \mathbf{r}_{k}-\mathbf{A}_{c} \tilde{\mathbf{X}}_{k-1}^{+} \mathbf{r}_{k-1} \\
& =\mathbf{h}_{k}^{c}+\tilde{\mathbf{X}}_{k}^{+} \mathbf{w}_{k}-\mathbf{A}_{c} \mathbf{h}_{k-1}^{c}-\mathbf{A}_{c} \tilde{\mathbf{X}}_{k-1}^{+} \mathbf{w}_{k-1} .
\end{aligned}
$$

According to (12) we also have: $h_{k}^{c}=\mathbf{A}_{c} h_{k-1}^{c}+\tilde{C} v_{k-1}$, thus the last form of the previous equation can be further written as $\mathbf{S}_{k}=$ $\overline{\mathbf{C}} \mathbf{v}_{k-1}+\overline{\mathbf{X}}_{k}^{+} \mathbf{w}_{k}-\mathbf{A}_{c} \overline{\mathbf{X}}_{k-1}^{+} \mathbf{w}_{k-1}$. Going back to (15), using the latter form of $\mathbf{S}_{k}$ and taking the expectation will lead to:

$$
\tilde{\mathbf{C Q}} \tilde{\mathbf{C}}^{H}=\mathbf{B}_{1}^{k}-\tilde{\mathbf{X}}_{k}^{+} \mathbf{R}\left(\tilde{\mathbf{X}}_{k}^{+}\right)^{H}-\mathbf{A}_{c} \tilde{\mathbf{X}}_{k-1}^{+} \mathbf{R}\left(\tilde{\mathbf{X}}_{k-1}^{+}\right)^{H} \mathbf{A}_{c}^{H},
$$

where $\mathbf{B}_{1}^{k}$ is estimated as follows:

$$
\begin{aligned}
\hat{\mathbf{B}}_{1}^{k}=\frac{1}{k-3} \sum_{n=3}^{k} & {\left[\left(\check{\mathbf{X}}_{n}^{+} \mathbf{r}_{n}-\mathbf{A}_{c} \check{\mathbf{X}}_{n-1}^{+} \mathbf{r}_{n-1}\right)\right.} \\
& \left.\left(\ddot{\mathbf{X}}_{n}^{+} \mathbf{r}_{n}-\mathbf{A}_{c} \ddot{\mathbf{X}}_{n-1}^{+} \mathbf{r}_{n-1}\right)^{H}\right] .
\end{aligned}
$$

In a mobile system the spatial correlation matrix $\tilde{\mathbf{C}}$ may be also time varying due to moving scatterers or the mobile terminal [8]. The recursive update of $\mathrm{CQC} \bar{C}^{H}$ in (17) allows also the tracking of 


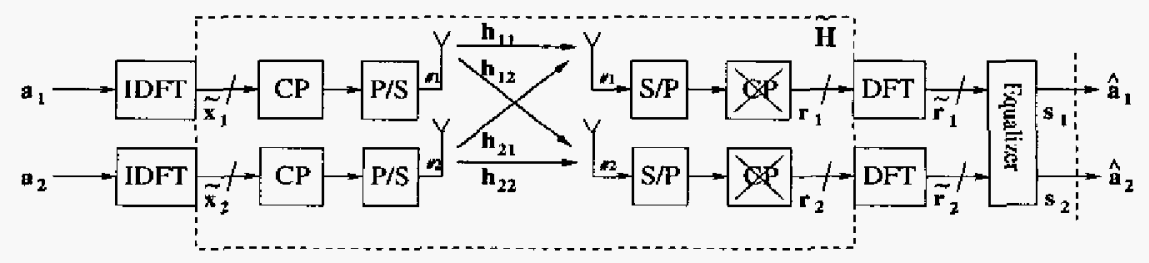

Fig. 1. $2 \times 2$ MIMO OFDM transmission.

the correlation matrix. However, an adaptive update with forgetting factor may be needed in equation (18).

The $\mathrm{KF}$ parameter acquisition stage is using known training symbols before actual data is transmitted. After this stage, the algorithm may switch to decision directed mode, where received symbols are equalized using MMSE techniques as presented in [13].

\section{Simulations}

In this section we briefly present the channel measurement setup, the simulation setup and the results obtained using the parameter estimation method. We want to emphasis that measured MIMO channels are used in MIMO OFDM simulations (i.e. no actual MIMO OFDM transmission took place in channel sounding).

\section{A. Measurement setup}

The channel measurements used in this paper took place at the Institute of Communications and Radio-Frequency Engineering, Vienna University of Technology. They were performed together with Elektrobit, using the Elektrobit PropSound channel sounder. We measured the impulse response between each transmit and receive antenna at a center frequency of $2.4 \mathrm{GHz}$ using a total measurement bandwidth of $200 \mathrm{MHz}$, which gives a delay resolution of 5 ns. At transmit side an 8-e]ement circular array of monopoles with 7 elements on the circle and one in the center and at receive side a 16element $4 \times 4$ rectangular patch array were used. Both arrays had an inter-element spacing of $0.5 \lambda$ at $2.55 \mathrm{GH} z$. During the measurement the receiver has been moved (with pedestrian speed), leading to a time-varying MIMO channel.

\section{B. Simulation setup}

In the simulations, we consider a $2 \times 2$ MIMO system, therefore we use only two transmit and two receive antennas from the available data. Since we have at the transmit side an 8-element circular array, we select different transmit schemes by using different pairs of transmit-receive antennas (i.e. the transmit antennas are changed between the 8 elements while the receive antennas are also changed between the 16 elements, hence we obtain 128 scenarios). From the measured impulse responses, we extract a bandwidth of $\mathrm{B}=1 \mathrm{MHz}$ which consists of two channel taps. The second tap has much lower power than the first one, therefore it was considered as noise. Hence, only one channel tap has been considered per MIMO branch. In order to create a channel suitable for our simulations, we interpolate between the snapshots that were measured every $37 \mathrm{~ms}$ such that the coherence time of the channel equals the OFDM symbol duration. Considering that the OFDM system has $N=32$ subcarriers, we expect that the channel impulse response has a length of less than $1 \mu s$, hence the total OFDM symbol duration becomes $33 \mu s$.

Since we consider that this is a parameter acquisition stage, the modulation used is BPSK. The number of subcarriers can be adjusted according to the size of the state vector. We recall that the state size is $m n L$, in our simulation $2 \times 2 \times 1=4$. The requirement is that the size of the observations is larger than that of the state. In MIMOOFDM the observation vector is of dimension $n N$, where $\mathrm{N}$ is the number of subcariers, $n N \gg m n L$.

The observation noise covariance matrix and state transition matrix have to be known when applying the estimator proposed in (17). However, both quantities can be also estimated [4], [5].

\section{Simulation results}

In the simulations presented in this paper, we deal with measured data where the channel was non-stationary. Therefore, the changes in the spatial structure of the channel has to be taken into account since this directly influences the estimation stage proposed in this paper. In other words, we have to know how fast the spatial correlation is changing such that we are able to te-cstimate the parameters of the state space model accordingly.

A new metric for characterizing the spatial non-stationarity of MIMO channels has been introduced in [8]. There, investigations based on the same set of measurements (as used in this paper) are presented. Based on the findings presented in [8], the parameter estimation is performed using time intervals of 2 seconds, which is the time interval where the spatial structure can be assumed to be relatively constant. Hence, our parameter estimation is performed in an interval where the channel is considered stationary.

Since the process noise covariance matrix is not known, a convergence of the estimated parameters to the true ones cannot be depicted. However, the estimated parameters do converge to steady state values. The Mean Square Error (MSE) of channel estimation using estimated model parameters is depicted in Fig. 2.

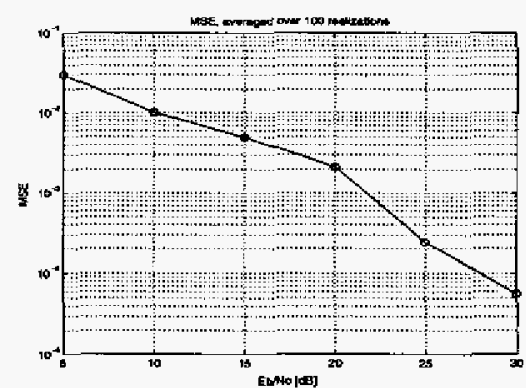

Fig. 2. MSE for channel estimates using estimated state-space model parameters computed with (17).

The system performance is characterized by bit error rate (BER). We have studied the raw BER using the true channel state information (CSI) and channel estimated using the approach described in this paper, Fig. 3.

The track of the real and imaginary parts of the channels are depicted in Figures 4 and 5 where $\mathrm{KF}$ is using the estimated correlation as shown in (17). Dash lines represents true values, continuous line represents estimated values. Note that good tracking 


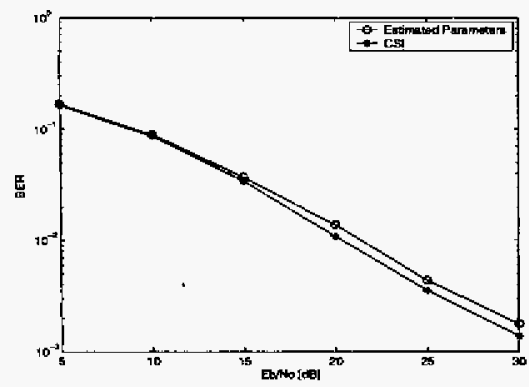

Fig. 3. BER of the receiver when channel estimation is performed using true and estimated state-space parameters.

performance is obtained due to good estimates of $\overline{\mathbf{C}} \mathbf{Q} \overline{\mathbf{C}}^{H}$ that lead to close to optimal performance of KF.
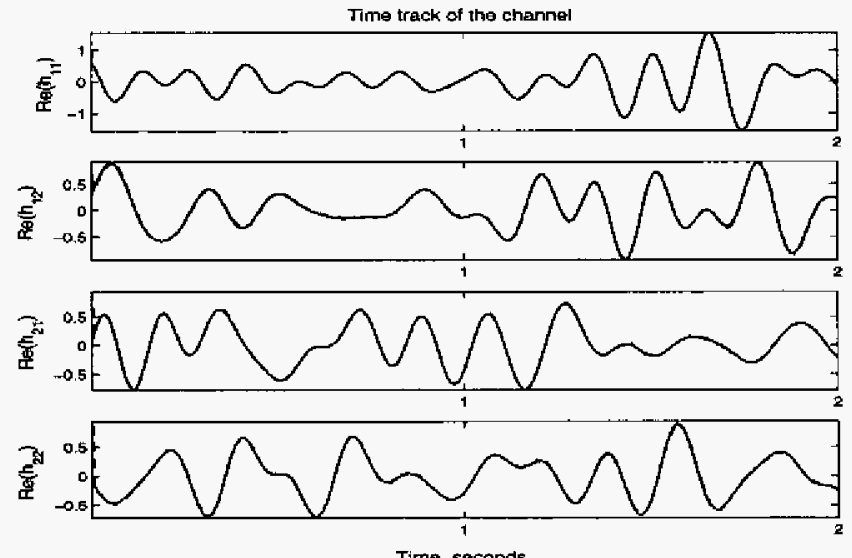

Fig. 4. Time track of real part of the channel using estimated model parameters. The dash and continuous lines overlap almost perfectly due to the good parameter estimation.
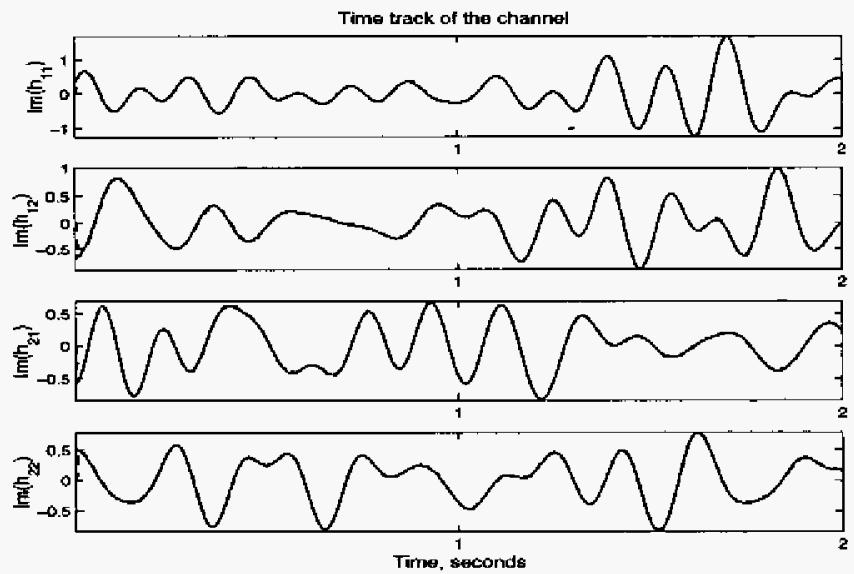

Fig. 5. Time track of imaginary part of the chamel using estimated model parameters. The dash and continuous lines overlap almost perfectly due to the good parameter estimation.

\section{CONCLUSION}

In this paper, channel parameter estimation and channel estimation and tracking experiments for mobile MIMO-OFDM systems have been carried out for MIMO measured channels. The current investigations have been limited to a low spatial resolution, i.e. to a $2 \times 2$ system. We have shown that good channel estimation can be performed when reliably estimating the state-space parameters. Future work includes studying systems with more antennas and using an advanced MIMO channel model [16] that is especially useful for systems with higher spatial resolution.

\section{ACKNOWLEDGMENTS}

The authors would like to thank Prof. Ernst Bonek for providing the MIMO measurements taken at the Institute of Communications and Radio-Frequency Engineering. Furthermore, the authors would like to thank Hüseyin Özcelik for helping a lot with the measurements and also Elektrobit for doing the measurements with us.

\section{REFERENCES}

[1] "IST-1999-11729 METRA Project", available at http: //www . ist-metra.org/

[2] E. Bonek, H. Özcelik, M. Herdin, W. Weichselberger, W. Wallace. "Deficiencies of a Popular Stochastic MIMO Radio Channel Model". International Symposium on Wireless Personal Multimedia Communications (WPMC'03), Oct. $19-22,2003$, Yokosuka, Japan.

[3] D. Chizhik, J. Ling, P. W. Wolniansky, R. A. Valenzuela, N. Costa, K. Huber, "Multiple-Input-Multiple-Output Measurements and Modeling in Manhattan", IEEE J. on Selected Areas in Comm., vol. 21, no. 3, 2002.

[4] M. Enescu, M. Sirbu, V. Koivunen, "Recursive Estimation of Noise Statistics in Kalman Filter Based MIMO Equalization", URSI General Assembly, CD-ROM proceedings, 2002, available at http://wooster hut.fi/statsp/publications . html

[5] M. Enescu, V. Koivunen, "On the estimation of state transition matrix and noise statistics in state-space models", Vehicular Technology Conference, 2002. Proceedings. VTC 2002-Fall. 2002 IEEE 56th, Volume: 4, 2002 Page(s): 2192 -2196.

[6] D. Gesber, and J. Akhtar, "Breaking the barriers of Shannon's capacity: An overview of MIMO wireless systems", Telektronikk Telenor J., Jan. 2002.

[7] A. Goldsmith, S. A. Jafar, N. Jindal, S. Vishwanath, "Capacity Linits of MMO Channels", IEEE J. on Selected Areas in Comm., vol, 21, no. 5, pp. 684-702, 2003.

[8] M. Herdin, E. Bonek, "A MIMO Correlation Matrix based Metric for Characterizing Non-Stationarity", in The IST Mobile and Wireless Communications Summit, Lyor, 2004.

[9] I. P. Kermoal, L. Schumacher, K. I. Pedersen, P. E. Mogensen, F. Frederiksen, "A Stochastic MIMO Radio Channel Model With Experimental Validation", IEEE J. on Selected Areas in Comm., 20(6):1211-1225, 2002.

[10] C. Komninakis, C. Fragouli, A. H. Sayed, and R.D. Wesel, "Multi-input multi-output fading channel tracking and equalization using Kalman estimation" Signal Processing, IEEE Transactions on, vol.50, no.5, pp. $1065-1076,2002$.

[11] K. A. Myers, B. D. Tapley "Adaptive Sequential Estimation with Unknown Noise Statistics", IEEE Transactions on Automatic Control, vol. AC-21, No.4, Aug. 1976, pp. 520-523.

[12] A. J. Paulraj, D. A. Gore,R, U. Nabar, H. Bolcskei, "An Overview of MIMO CommunicationsA Key to Gigabit Wireless" Proceedings of the IEEE , vol. 92 , no. 2 , Feb. 2004, pp: $198-218$.

[13] T. Roman, M. Enescu, V. Koivunen, "Time-domain method for tracking dispersive channels in MIMO OFDM systems", ICASSP, Vol. 4, pp. 393 $-396,2003$

[14] D-S. Shiu, G. J. Foschini, M. J. Gans, J. M. Kahn, "Fading Correlation and Its Effects on the Capacity of Multielement Antenna Systems", IEEE Trans. on Comm., 40(3):502-513, 2000

[15] M.K. Tsatsanis, G.B. Giannakis, G. Zhou, "Estimation and Equalization of fading channels with random coefficients", Signal Processing, 53, pp.: 211-229, 1996.

[16] W. Weichselberger, H. Özcelik, M. Herdin, E. Bonek, "A Novel Stochastic MIMO Channel Model and its Physical Interpretation", International Symposium on Wireless Personal Multimedia Communications (WPMC'03), Oct. $19-22,2003$, Yokosuka, Japan. 